\title{
NEUROCIENCIAS PARA LA GESTIÓN DE LOS RECURSOS HUMANOS Y COMPORTAMIENTO ORGANIZACIONAL
}

\section{NEUROSCIENCE RESOURCES FOR HUMAN RESOURCES AND ORGANIZATIONAL BEHAVIOR MANAGEMENT}

\section{Carlos Bancayán Oré y Carlos Julián Dávila Chávez}

\section{Resumen}

Siendo el trabajo una actividad humana que se da a través de una dependencia remunerativa y que se ejecuta en contexto organizacional que demanda a los colaboradores, el uso de conductas específicas, complejas y especializadas, donde para las empresas es una necesidad el identificar, evaluar, capacitar y desarrollar a sus colaboradores, las Neurociencias pueden aportar un enfoque que puede llegar a dar comprensión y una explicación objetiva de cómo aquella conducta que se produce en las relaciones laborales, de gestión y dirección tienen base neuroanatómicas funcionales en la corteza prefrontal, que al ser evaluada por la neuropsicología podría dar información neurocientífica de la conducta laboral y de sus correspondientes regiones neurobiológicas supramodales asociativas.

Palabras clave: Neurociencias; neuroanatómicas; neuropsicología; relaciones laborales; comportamiento organizacional.

\section{Abstract}

Being work a human activity, that occurs through a remunerative dependency and that executes in an organizational context that demands from the collaborators, the use of specific, complex and specialized behaviors, whic for the companies, is a need to identify, evaluate, train and develop their collaborators, can provide an 
approach that can give understanding and an objective explanation of how that behavior that occurs in labor relations, management and administration have functional neuroanatomical basis the prefrontal cortex that being evaluated by neuropsychology could give neuroscientific information of the work behavior and its corresponding neurobiological supramodal associative regions.

Key words: Neurosciences; neuroanatomical; Neuropsychology; labour relations; organizational behavior.

EL ESTUDIO DE LA CONDUCTA HUMANA EN LAS RELACIONES LABORALES

La búsqueda para comprender la conducta del ser humano ha devenido en el interés de diversas ciencias a fin de proveer un mejor entendimiento y conocimiento del comportamiento, sus diversas áreas y actividades.

A fines del siglo XIX, las Ciencias Administrativas buscaban dar explicación a los factores productivos dentro de las actividades industriales, comerciales y laborales.

A principios del siglo XX, una de las metas más relevantes en los primeros estudios sobre la comprensión y orientación de la conducta en el ámbito laboral, se dio debido a que se buscaba que el individuo tuviera la mejor predisposición y desarrollo comportamental en sus actividades de trabajo, a través de análisis factoriales estadísticos de las características personales y demanda ocupacional, elaborándose así las primeras pruebas psicométricas en la Universidad de Stanford.

La búsqueda de poder gestionar las relaciones de trabajo, más allá de una compensación monetaria, se produjo como consecuencia de la comprensión de la importancia del comportamiento grupal, sentido de pertenencia, identidad, motivación, autorrealización, así como de las respectivas competencias organizacionales capaces de generar una mejora en la eficiencia de los recursos, productividad y desarrollo comercial.

Sin embargo, el recuento no fue totalmente concluyente, pues los esfuerzos por tratar de entender lo descrito aun no culmina, y se requiere de una interpretación más precisa, apoyándonos en nuevas disciplinas que permitan entender el problema.

\section{NEUROCIENCIA}

La Neurociencia se desarrolla dentro del campo del estudio del cerebro, mediante el estudio multidisciplinar del sistema nervioso, los procesos neurobiológicos y los mentales.

El abordaje neurocientifico busca unificar el entendimiento de la relación de la actividad cerebral con la conducta humana.

Los aportes de diversas disciplinas brindan una concepción integral y novísima del ser humano, al dar a conocer cómo las funciones del sistema nervioso producen la conciencia, la cognición, la afectividad, la flexibilidad cerebral, etc.

La neurociencia contiene los es- 
tudios neuroanatomicos, químicos $\mathrm{y}$ patológicos, del neurodesarrollo y los procesos neurofuncionales, permitiendo de esta manera un acercamiento a los procesos mentales superiores, la personalidad, la conducta social y, por tanto, la conducta laboral y organizacional.

Si bien las Neurociencias contienen conocimientos propios del cerebro, cada una de ellas contiene una finalidad, las cuales se puede tipificar como conductuales y no conductuales. Las primeras se interesan en los procesos mentales y sus bases neurales, en tanto que las segundas se interesan en las estructuras cerebrales, pero sin profundizar en los procesos cognitivos o mentales superiores que producen.

Es necesario tener en cuenta que la actividad del sistema nervioso central tiene como traducción última a la conducta.

A su vez, a nivel seudocientífico, se puede comprender que la cognición como proceso superior del cerebro es la produce el pensamiento, el lenguaje, la percepción, el movimiento intencional, la inhibición, el control emocional, el planeamiento, etc. A través de técnicas invasivas y no invasivas se ha podido graficar y topográficamente señalar cuáles son las bases de cada una de las funciones neurocompartamentales.

Así, la imagenología, o neuroimagen funcional, ha dispuesto la traducción inmediata de los procesos neurológicos de un proceso mental, de tal forma que al realizar una actividad cognitiva, se pueda saber la localiza- ción, estado y funcionamiento de una estructura cerebral.

Aquella parte de la neurociencia que pueda realizar la actividad anteriormente mencionada, debe ser una neurociencia comportamental; correspondiendo a la neuropsicología o neurología de la conducta (Benton, 1971), que tiene por objeto de estudio la madurez clínica y el desarrollo histórico científico (la cual contiene la capacidad de realizar estudios neurofisiológicos, imaginológicos y neuropsicológicos), que permiten describir la expresión neuroanatomía funcional y comportamental.

\section{NEUROPSICOLOGÍA}

El desarrollo de la Neuropsicología y su búsqueda de poder alcanzar información sobre las bases cerebrales y funcionales en las que se basa la conducta humana, llevó a diferentes tipos de estudios y teorias sobre el funcionamiento cerebral y comportamental.

Según Golberg (2001), las funciones más complejas del ser humano se encuentran a nivel de la corteza prefrontal, que generan el control, la regulación y la planeación eficiente de la conducta humana. Para Lezak (2004), el desarrollo neuroconductal permite que los sujetos puedan ejecutar actividades independientes, eficientes $\mathrm{y}$ útiles.

Para Portellano (2005), el estudio de las funciones mentales superiores, que topográficamente se encuentran en el lóbulo frontal, evidencia que la corteza prefrontal contiene el fun- 
cionamiento de la planificación de la conducta. La neuroanatomía de los lóbulos frontales contiene una organización funcional localizada en las regiones de la corteza prefrontal, que se divide en tres regiones principales, cada una de las cuales presenta una funcionalidad particular, según Flores y Ostrosky (2008).

Para Ardila y Ostrosky (2012), cuando se encuentra un daño o disfunción en la corteza orbito frontal se producen cambios neurocomportamentales que generan una merma o pérdida de la adecuación social, del lenguaje, de la regulación de la conducta y de las emociones, de insensibilidad hacia los otros y de desinterés por los sucesos actuales.

A su vez, el desarrollo de esta especialidad tiene sus orígenes en el desarrollo de la neurología. El ámbito de aplicación de esta ciencia se daba por un método médico inductivo, semiológico y cualitativo, que al consolidarse posibilitó la aparición de diferentes tipos de métodos Neuropsicológicos, como un proceso de evaluación neurocientífica, utilizando diversas técnicas para explicar el comportamiento humano.

\section{NEUROPSICOLOGÍA Y COM- PORTAMIENTO LABORAL}

El interés por conocer y evaluar neurocientificamente conducta humana para obtener una explicación y correlato neurofuncional, más aún la capacidad de investigar como el desarrollo de la actividad laboral, se mani- fiesta dentro de las funciones neuropsicológicas de los trabajadores.

El desarrollo de la conducta humana supeditada a una relación de trabajo demanda una serie de actividades que están estipuladas y son de obligatorio cumplimiento a nivel laboral y jurídico legal, por tanto, se encuentran a la búsqueda de un resultado específico dentro de un diseño ocupacional.

La búsqueda de que todos los trabajadores, logren ejecutar actividades que se den dentro de las propias dinámicas que confluyen en la creación y sustentación de los servicios, a través de competencias que logren el éxito de cada puesto de trabajo que se reditúe con éxito para la empresa y su competitividad en el mercado, donde desarrolle sus actividades comerciales, aun es un tema pendiente.

Hoy en dia los trabajadores constituyen un grupo muy importante de la población y su capacidad conductual es aquella que le permite seguir dentro de actividades laborales, en la cual se generan efectos favorables como el mantenimiento de una sociedad económicamente productiva, desarrollo de empresarial y la viabilidad del proyecto de vida de las personas debido a los efectos que produce una relación de trabajo como la remuneración, la identidad, el desarrollo de la personalidad y goce de beneficios de los derechos sociales que devienen del trabajo.

No cabe duda que la organización, es el evidente espacio del ambiente de trabajo, las condiciones laborales, son 
elementos constitutivos, para el desarrollo de las actividades dentro de una organización.

Por otro lado, es evidente que sin una capacidad conductual alineada al puesto de trabajo, no se podrán generar los resultados adecuados al diseño ocupacional y más aun de la estructura productiva de la organización.

El desarrollo de las neurociencias permite entender que la conducta humana tiene una base cerebral, estas se dan tanto en estructuras, sistemas, circuitos y esquemas de interconexión de la cual deviene diferentes tipos de funcionamiento específico de áreas cerebrales y sus respectivas interacciones.

La cognición y la conducta humana, tienen por tanto la capacidad de ser estudiados, localizados y evaluados, reflejando así la capacidad de hablar de un neurocomportamiento, el cual es la relación del cerebro y la conducta, y llegar a un más a la especificidad de aquellas funciones, las cuales generan una conductas complejas, que hacen a los seres humanos funcionales dentro de las actividades sociales, educativas, afectivas y laborales.

\section{NEUROPSICOLOGIA Y COMPOR- TAMIENTO ORGANIZACIONAL}

A nivel neuropsicológico, el estudio del cerebro se desarrolla a través las estructuras neuroarquitectónicas, o áreas cerebrales específicas.

Las funciones mentales superiores se ubican en la corteza prefrontal y sus regiones quienes posibilitan las conductas morales, sociales, cognitivas superiores y funciones ejecutivas del más alto nivel de análisis y desarrollo humano, tanto para las relaciones humanas y a su vez las competencias organizacionales.

La identificación y estudio neurológico, nos lleva a poder formular la importancia de como las funciones de las áreas cerebrales y sus respectivo estudio neuropsicológico nos da la disposición de información relevante de la conducta y por tanto permitiría el identificar y encontrar métodos neurocientificos relevantes y necesarios para selección, capacitación y gestión en el Comportamiento Organizacional.

A su vez la búsqueda de poder disponer de procedimientos, técnicas, herramientas para medir o reflejar conductas complejas que puedan reflejar como actuaciones laborales donde se confieren sinérgicamente recursos económicos, administrativos y relacionales para la ejecución de lo estipulado a nivel organizacional, que se representa en los productos y servicios, que dotan a las empresas de una constante generación de insumos que se manifiestan en demandas para el crecimiento corporativo. Las distintas formas en las que hoy pueden llegar a estipularse las condiciones laborales, los distintos regímenes laborales y como estos disponen del colaborador interno hacen que variables organizacionales como las relaciones interpersonales, valores organizaciones, habilidades blandas, toma de decisiones, resolución de conflictos, habili- 
dades directivas dentro del entorno laboral son hoy competencias deseadas dentro de la conducta organizacional, como es el caso de la gestión del talento humano, comportamiento organizacional y relaciones de trabajo productivas y rentables para las corporaciones.

\section{NEUROPSICOLOGÍA PARA LA GESTIÓN DE LOS RECURSOS HU- MANOS}

A nivel neuroanatómico funcional, se pueden estructurar diferentes áreas cerebrales que son identificables topográficamente y que amparan las funciones mentales superiores; en ellas se apoyan las competencias laborales dentro de la Gestión de los Recursos Humanos, siendo la corteza prefrontal muy relevante para su estudio.

Es posible evidenciar, por tanto, que las regiones neuroanatómicas y sus consecuentes funciones neuropsicológicas son sumamente relevantes para la Gestión de los Recursos Humanos, debido a que lo que deviene de las mismas son las conductas que capitalizan las relaciones laborales; más aún, son las que posibilitan que puedan darse las instancias para el desarrollo de la empresa a través de las conductas de sus trabajadores, una planificación de desarrollo, así como el desenvolvimiento de los procesos empresariales de departamentalización, descentralización, empoderamiento y gestión del conocimiento. Además posibilitan decisiones que no afecten el desarrollo económico, comercial e institucional, promoviendo la prevención de delitos y crimines dentro de la esfera laboral.

\section{CORTEZA ORBITOFRONTAL Y DESARROLLO DE LAS RELACIONES LABORALES}

La Corteza Orbitofrontal posibilita que la conducta humana adopte medidas capaces de inhibir conductas lesivas, impulsivas, antisociales y criminales. Estas, a su vez, se extrapolan en el ámbito organizacional, dentro de las afectaciones producidas por los trabajadores en una instancia laboral, que van desde falta de modulación de conducta dentro del entorno de trabajo, impulsividad en la gestión de departamentos y relaciones con pares, afectación a las políticas y normas que rigen los reglamentos internos de trabajo, conductas lesivas como la disposición irregular de bienes patrimoniales, dinerarios y no dinerarios de la empresa. Asimismo, permiten el control de acciones de nivel antisocial en la gestión de personal, como hostigamiento laboral, liderazgo y dirección coercitiva, además de conductas criminales dentro de las relaciones de subordinación de trabajo, como el acoso sexual, la apropiación licita, los despidos indirectos, el daño moral, el daño psicológico y la violencia de género, como lo prescribe la legislación laboral. Esto es muy importante, porque hoy la gestión de los Recursos Humanos demanda una estricta adecuación al ordenamiento jurídico actual, que reconoce a la sujeción laboral como una posición de vulnerabilidad 
a quien bajo el principio de dirección y fiscalización puede vulnerar derechos inherentes a quien está bajo su subordinación.

\section{CONCLUSIONES}

- La Neurociencia posibilita dar una comprensión del comportamiento en el ámbito laboral, organizacional y de los Recursos Humanos, a su vez de proveer una metodología para el análisis y estudio de la relación entre el cerebro y la conducta a través de la Neuropsicología.

- Se ha evidenciado que las regiones neuroanatómicas y sus funciones neuropsicológicas son muy relevantes para la Gestión de los Recursos Humanos, debido a que lo que deviene de ella son las conductas que capitalizan el comportamiento laboral.

- También se puede evidenciar cómo a través del estudio de la corteza prefrontal se posibilita la capacidad del desarrollo de las competencias organizacionales. Las competencias organizacionales pueden proveer una conducta que posibilite la motivación, el trabajo en equipo, la comunicación y el liderazgo, lo que luego pueda reflejarse en la percepción del clima organizacional y relaciones interpersonales.

- La Gestión de los Recursos Humanos necesita de una dirección que pueda planificar actividades y procesos de la empresa a través de conductas de sus jefes y gerentes, además de prever cómo las mismas no afecten el desarrollo económico, comercial e institucional.

- Las relaciones laborales permiten posicionarse en una capacidad de prever conductas lesivas y promover las conductas adecuadas para el desarrollo de la fuerza de trabajo y el capital, donde no se comentan faltas éticas, malas prácticas laborales, delitos y crimenes dentro de la esfera laboral. 


\section{REFERENCIAS BIBLIOGRÁFICAS}

Ardila, A. (2013). Funciones Ejecutivas (Guía de evaluación). Miami: Florida Internacional University.

Ardila, A \& Ostrosky-Solis, F. (2012). Guía para el Diagnóstico Neuropsicológico. Miami: Florida International University.

Ardila, A., Arocho-Llantín, J.L., Labos, E. \& Rodriguez- Irizarry, W. (2015). Diccionario de Neuropsicología. Ciudad: editorial

Buller, I. (2010). "Evaluación Neuropsicológica Efectiva de la Función Ejecutiva", en: Revista Neuropsicología, Neuropsiquiatria y Neurociencias, 4 (1), 63-86.

Benton, AL. (1971). Introducción a la neuropsicología. Barcelona: Fontanella.

Flores, J. \& Ostrosky- Shejet, F. (2012). Desarrollo Neuropsicológico de los Lóbulos Frontales y Funciones ejecutivas. México: Manual Moderno

Moraine, Paula (2014). Las funciones ejecutivas del estudiante. Madrid: Narcea.

Sigman, Mariano (2015). La vida secreta de la mente: nuestro cerebro cuando decidimos, sentimos y pensamos. Buenos Aires: Debate.

Tirapu Ustárroz, J., García-Molina, A., Ríos Lago, M. \& Ardila, A. (2012). Neuropsicología de la corteza prefrontal y las funciones ejecutivas. Barcelona: Editorial Viguera.

Tirapu, J., Luna, P. (2008). Neuropsicología de las Funciones Ejecutivas. Manual de Neuropsicología. Barcelona: Viguera Editores. 